Material and Spatial Motion Problems in Nonlinear Electro- and Magneto-elastostatics

D.K. Vu and P. Steinmann

Mathematics and Mechanics of Solids 2010 15: 239 originally published online 11 March 2009

DOI: $10.1177 / 1081286508098583$

The online version of this article can be found at:

http://mms.sagepub.com/content/15/2/239

Published by:

(S)SAGE

http://www.sagepublications.com

Additional services and information for Mathematics and Mechanics of Solids can be found at:

Email Alerts: http://mms.sagepub.com/cgi/alerts

Subscriptions: http://mms.sagepub.com/subscriptions

Reprints: http://www.sagepub.com/journalsReprints.nav

Permissions: http://www.sagepub.com/journalsPermissions.nav

Citations: http://mms.sagepub.com/content/15/2/239.refs.html

>> Version of Record - Feb 24, 2010

OnlineFirst Version of Record - Mar 11, 2009

What is This? 


\title{
Material and Spatial Motion Problems in Nonlinear Electro- and Magneto-elastostatics
}

\author{
D. K. VU \\ P. STEINMANN \\ Friedrich-Alexander University of Erlangen-Nuremberg, Erlangen, Germany
}

(Received 10 July 2008; accepted 5 September 2008)

\begin{abstract}
In this paper, material and spatial motion problems of the coupled nonlinear problem of electroand magneto-elastostatics are discussed in the context of non-potential loading where mechanical loads are not assumed to be derived explicitly from some potential. A virtual work approach is used to derive the corresponding balance equations and boundary conditions of the material motion problems.
\end{abstract}

Key Words: nonlinear coupling, nonlinear elasticity, electricity, magnetism, material forces

\section{INTRODUCTION}

In the past few years, the potential application of electro- and magneto-elastic materials in developing artificial muscles [1-3], has lead to considerable research effort on the modeling and simulation of these materials, see for example [4-22]. Besides modeling and simulation, defect analysis of electro- and magneto-elastic materials, especially by the concept of configurational forces, is also a subject of important concern and was examined in many works, e.g [23-31]. The concept of configurational forces in nonlinear electro- and magneto-elastostatics was revisited in a recent study [32], where the balance equations of linear momentum (or the balance equations of linear momentum of the spatial motion problem, as they are called in the parlance of configurational mechanics) and the corresponding boundary conditions are transformed into some appropriate forms (material motion forms) and in an appropriate continuum mechanical setting (the material setting). Since the governing equations and variational formulations of the nonlinear electro- and magneto-elastostatic problems were derived by an energy approach, the application of these formulations are limited by the assumption made in the study that mechanical loads can be derived explicitly from some potential. In order to overcome this limitation, material and spatial motion problems in nonlinear electro- and magneto-elastostatics are examined in this work by a virtual work approach.

(C) The Author(s), 2010. Reprints and permissions:

http://www.sagepub.co.uk/journalsPermissions.nav 


\section{SPATIAL AND MATERIAL MOTION PROBLEMS}

To facilitate the derivation process, some basic notations in the case of nonlinear elastostatics are first recalled. We examine here a body with its material (reference) configuration $\mathcal{B}_{0}$ in the absence of electric and magnetic fields and mechanical loads. The spatial (current) configuration of the body is denoted by $\mathcal{B}_{t}$. For the static cases, in the spatial motion problem, the position vector $\mathbf{x}$ of a point in the spatial configuration $\mathcal{B}_{t}$ is described by the nonlinear spatial motion map $\mathbf{x}=\boldsymbol{\varphi ( X )}$ and the deformation is characterized by the spatial motion deformation gradient $\mathbf{F}=\nabla_{\mathbf{X}} \boldsymbol{\varphi}$ wherein $\nabla_{\mathbf{X}} \boldsymbol{\varphi}$ denotes $\partial \varphi_{i} / \partial X_{j}$. In the material motion problem, the position vector $\mathbf{X}$ of a point in the material configuration $\mathcal{B}_{0}$ is described by the nonlinear material motion map $\mathbf{X}=\boldsymbol{\Phi}(\mathbf{x})$ and the deformation is characterized by the material motion deformation gradient $\mathbf{f}=\nabla_{\mathbf{x}} \boldsymbol{\Phi}$. For a conservative mechanical system, the elastic material response is characterized by some internal potential energy densities per unit volume $W_{0 \mathbf{F}}, W_{0 \mathbf{f}}$ in material configuration or $W_{t \mathbf{F}}, W_{t \mathbf{f}}$ in spatial configuration:

$$
\begin{array}{ll}
W_{0 \mathbf{F}}=W_{0 \mathbf{F}}(\mathbf{F} ; \mathbf{X}) ; & W_{0 \mathbf{f}}=W_{0 \mathbf{f}}(\mathbf{f}, \boldsymbol{\Phi}) \\
W_{t \mathbf{F}}=W_{t \mathbf{F}}(\mathbf{F} ; \mathbf{X}) ; & W_{t \mathbf{f}}=W_{t \mathbf{f}}(\mathbf{f}, \mathbf{\Phi})
\end{array}
$$

such that $\left.W_{0 \mathbf{F}}\right|_{\mathbf{F}, \mathbf{X}}=\left.W_{0 \mathbf{f}}\right|_{\mathbf{f}, \Phi}$ and $\left.W_{t \mathbf{F}}\right|_{\mathbf{F}, \mathbf{X}}=\left.W_{t \mathbf{f}}\right|_{\mathbf{f}, \boldsymbol{\Phi}}$

\section{NONLINEAR ELASTOSTATICS}

\subsection{Spatial Motion Problem}

Consider the spatial problem in nonlinear elasticity. In reference to the deformed configuration $\mathcal{B}_{t}$, the balance equation of linear momentum can be derived by varying the spatial placement an amount $\delta \mathbf{x}$ while keeping the material placement fixed. If we denote the applied body force by $\mathbf{b}_{t}$ and the applied surface traction by $\mathbf{t}_{t}$, the principle of virtual work states that

$$
\mathrm{W}_{i n t}-\mathrm{W}_{e x t}=0
$$

where $\mathbf{W}_{\text {int }}$ and $\mathbf{W}_{\text {ext }}$ are the internal and external virtual work corresponding to the variation $\delta \mathbf{x}$ taken at fixed material placement $\delta \mathbf{X}=\mathbf{0}\left(\right.$ denoted $\left.\delta \mathbf{x}=\delta_{\mathbf{X}} \boldsymbol{\varphi}\right)$ :

$$
\mathbf{W}_{i n t}=\delta_{\mathbf{X}} \int_{\mathcal{B}_{t}} W_{t \mathbf{F}} \mathrm{d} v \quad \text { and } \quad \mathbf{W}_{e x t}=\int_{\mathcal{B}_{t}} \mathbf{b}_{t} \cdot \delta_{\mathbf{X}} \boldsymbol{\varphi} \mathrm{d} v+\int_{\partial \mathcal{B}_{t}} \mathbf{t}_{t} \cdot \delta_{\mathbf{X}} \boldsymbol{\varphi} \mathrm{d} s .
$$

The localization of (2) reads

$$
\nabla_{\mathbf{x}} \cdot \boldsymbol{\sigma}+\mathbf{b}_{t}=\mathbf{0} \quad \text { in } \mathcal{B}_{t} \quad \text { and } \quad \boldsymbol{\sigma} \cdot \mathbf{n}=\mathbf{t}_{t} \quad \text { on } \partial \mathcal{B}_{t}
$$


where $\nabla_{\mathbf{x}} \cdot\{\bullet\}$ denotes $\partial\{\bullet\}_{i j} / \partial x_{j}, \mathbf{n}$ is the outward pointing unit normal at the boundary $\partial \mathcal{B}_{t}$, and the spatial motion Cauchy stress tensor $\boldsymbol{\sigma}$ is defined as

$$
\boldsymbol{\sigma}:=W_{t \mathbf{F}} \mathbf{I}+\partial_{\mathbf{F}} W_{t \mathbf{F}} \cdot \mathbf{F}^{t}
$$

in which the upper script $t$ denotes transpose and $\mathbf{I}$ is the unit tensor.

In reference to the undeformed configuration $\mathcal{B}_{0}$, the counterparts of (3.1-2) have the form

$$
\mathbf{W}_{i n t}=\delta_{\mathbf{X}} \int_{\mathcal{B}_{0}} W_{0 \mathbf{F}} \mathrm{d} V \quad \text { and } \quad \mathbf{W}_{\text {ext }}=\int_{\mathcal{B}_{0}} \mathbf{b}_{0} \cdot \delta_{\mathbf{X}} \boldsymbol{\varphi} \mathrm{d} V+\int_{\partial \mathcal{B}_{0}} \mathbf{t}_{0} \cdot \delta_{\mathbf{X}} \boldsymbol{\varphi} \mathrm{d} S
$$

where we denote the applied body force and the applied surface traction in reference to $\mathcal{B}_{0}$ by $\mathbf{b}_{0}$ and $\mathbf{t}_{0}$, respectively:

$$
\mathbf{t}_{0}=J\left[\mathbf{N} \cdot \mathbf{C}^{-1} \cdot \mathbf{N}\right]^{1 / 2} \mathbf{t}_{t} \quad \text { and } \quad \mathbf{b}_{0}=J \mathbf{b}_{t}
$$

in which $J=\operatorname{det} \mathbf{F}, \mathbf{C}=\mathbf{F}^{t} \cdot \mathbf{F}$ and $\mathbf{N}$ is the outward pointing unit normal at the boundary $\partial \mathcal{B}_{0}$. With (6.1-2), the condition (2) leads to

$$
\nabla_{\mathbf{X}} \cdot \mathbf{P}+\mathbf{b}_{0}=\mathbf{0} \quad \text { in } \mathcal{B}_{0} \quad \text { and } \quad \mathbf{P} \cdot \mathbf{N}=\mathbf{t}_{0} \quad \text { on } \partial \mathcal{B}_{0}
$$

where $\mathbf{P}$ is the spatial motion Piola stress tensor:

$$
\mathbf{P}:=\partial_{\mathbf{F}} W_{0 \mathbf{F}}
$$

\subsection{Material Motion Problem}

In the material motion problem, if we vary the material placement an amount $\delta \mathbf{X}$, the new material configuration of the body is now $\mathcal{B}_{0}^{\delta}$. Corresponding to this configuration, under the application of the applied forces, we have a new spatial configuration $\mathcal{B}_{t}^{\delta}$. In order to keep the spatial placement unchanged, extra forces must be applied on the body to move each point on $\mathcal{B}_{t}^{\delta}$ back to its corresponding position on $\mathcal{B}_{t}$. If we denote the virtual work realized by these extra forces by $\mathrm{w}_{\text {conf }}$, then similar to (2) one obtains

$$
\mathrm{w}_{\text {int }}-\mathrm{w}_{\text {ext }}-\mathrm{w}_{\text {conf }}=0
$$

where $\mathbf{W}_{i n t}$ and $\mathbf{W}_{\text {ext }}$ are the internal and external virtual work corresponding to the variation $\delta \mathbf{X}$ taken at fixed spatial placement $\delta \mathbf{x}=\mathbf{0}$ (denoted $\delta \mathbf{X}=\delta_{\mathbf{x}} \boldsymbol{\Phi}$ ). In reference to spatial configuration, although the internal virtual work can be computed by 


$$
\mathbf{W}_{i n t}=\delta_{\mathbf{x}} \int_{\mathcal{B}_{t}} W_{t \mathbf{f}} \mathrm{d} v
$$

we cannot compute the external virtual work directly as in (3.2). However, we can assume that this virtual work can be written in the following form:

$$
\mathbf{W}_{\text {ext }}=\int_{\mathcal{B}_{t}} \mathbf{B}_{t}^{\text {ext }} \cdot \delta_{\mathbf{x}} \boldsymbol{\Phi} \mathrm{d} v+\int_{\partial \mathcal{B}_{t}} \mathbf{T}_{t}^{e x t} \cdot \delta_{\mathbf{x}} \boldsymbol{\Phi} \mathrm{d} s
$$

where $\mathbf{B}_{t}^{\text {ext }}=\mathbf{B}_{t}^{\text {ext }}\left(\mathbf{b}_{t}\right)$ is some body force and $\mathbf{T}_{t}^{\text {ext }}=\mathbf{T}_{t}^{\text {ext }}\left(\mathbf{t}_{t}\right)$ is some traction. Similarly, we can assume that $\mathbf{w}_{\text {conf }}$ can be written as

$$
\mathrm{w}_{\text {conf }}=\int_{\mathcal{B}_{t}} \mathbf{B}_{t}^{d} \cdot \delta_{\mathbf{x}} \boldsymbol{\Phi} \mathrm{d} v+\int_{\partial \mathcal{B}_{t}} \mathbf{T}_{t}^{d} \cdot \delta_{\mathbf{x}} \boldsymbol{\Phi} \mathrm{d} s
$$

where $\mathbf{B}_{t}^{d}$ is some body force and $\mathbf{T}_{t}^{d}$ is some traction. Note that in writing the virtual works in the above form, we have the freedom to choose $\mathbf{B}_{t}^{\text {ext }}, \mathbf{T}_{t}^{\text {ext }}, \mathbf{B}_{t}^{d}$ and $\mathbf{T}_{t}^{d}$ such that (10) is satisfied. For discussion about the case where external loads are derived from some potential, see for example [33].

By introducing (11), (12) and (13) into (10) one obtains the following the balance equation and boundary condition:

$$
\nabla_{\mathbf{x}} \cdot \mathbf{p}+\mathbf{B}_{t}=:-\mathbf{B}_{t}^{d} \quad \text { in } \mathcal{B}_{t} \quad \text { and } \quad \mathbf{p} \cdot \mathbf{n}-\mathbf{T}_{t}^{e x t}=: \mathbf{T}_{t}^{d} \quad \text { on } \partial \mathcal{B}_{t}
$$

where the material motion Piola stress tensor $\mathbf{p}$ and the material body force $\mathbf{B}_{t}$ are defined as

$$
\mathbf{p}:=\partial_{\mathbf{f}} W_{t \mathbf{f}} \quad \text { and } \quad \mathbf{B}_{t}:=\mathbf{B}_{t}^{\text {int }}+\mathbf{B}_{t}^{\text {ext }}
$$

in which the material body force $\mathbf{B}_{t}$ is decomposed into two parts: the first one is attributed to the change of internal energy density with respect to material placement $\mathbf{B}_{t}^{i n t}:=-\partial_{\boldsymbol{\Phi}} W_{t \mathbf{f}}$ and the second is due to the applied forces. From the definitions of the stress tensors $\boldsymbol{\sigma}$ and p, one has the relationships

$$
\mathbf{p}=W_{t \mathbf{f}} \mathbf{f}^{-t}-\mathbf{f}^{-t} \cdot \boldsymbol{\sigma} \quad \text { and } \quad \boldsymbol{\sigma}=W_{t \mathbf{f}} \mathbf{I}-\mathbf{f}^{t} \cdot \mathbf{p} .
$$

The introduction of (16.1) into (14.1), after some manipulation, leads to

$$
\nabla_{\mathbf{x}} \cdot \boldsymbol{\sigma}-\mathbf{f}^{t} \cdot \mathbf{B}_{t}^{e x t}=\mathbf{f}^{t} \cdot \mathbf{B}_{t}^{d} \quad \text { in } \mathcal{B}_{t}
$$

By comparing (4.1) and (17), one arrives at the definition

$$
\mathbf{B}_{t}^{e x t}:=-\mathbf{f}^{-t} \cdot \mathbf{b}_{t} \quad \text { and } \quad \mathbf{B}_{t}^{d}:=\mathbf{0}
$$


With the help of (4.2) and (16.1), the boundary condition (14.2) can be rewritten in the form

$$
W_{t} \mathbf{f}^{-t} \cdot \mathbf{n}-\mathbf{f}^{-t} \cdot \mathbf{t}_{t}=\mathbf{T}_{t}^{d}+\mathbf{T}_{t}^{e x t} .
$$

By noticing that the first term on the left-hand side of (19) can be interpreted as the flux of internal energy dissipated through the boundary $\partial \mathcal{B}_{t}$, this equation suggests the following definition of $\mathbf{T}_{t}^{d}$ and therefore $\mathbf{T}_{t}^{\text {ext }}$ :

$$
\mathbf{T}_{t}^{d}:=W_{t \mathbf{f}} \mathbf{f}^{-t} \cdot \mathbf{n} \quad \text { and } \quad \mathbf{T}_{t}^{e x t}:=-\mathbf{f}^{-t} \cdot \mathbf{t}_{t} .
$$

Turning to the material motion problem in reference to the material configuration $\mathcal{B}_{0}$, with respect to a variation $\delta \mathbf{X}$ of the material placement at $\delta \mathbf{x}=\mathbf{0}$, the internal virtual work is

$$
\mathrm{W}_{i n t}=\delta_{\mathbf{x}} \int_{\mathcal{B}_{0}} W_{0 \mathbf{f}} \mathrm{d} V
$$

In addition, the counterparts of (12) and (13) read

$$
\mathbf{W}_{\text {ext }}=\int_{\mathcal{B}_{0}} \mathbf{B}_{0}^{\text {ext }} \cdot \delta_{\mathbf{x}} \boldsymbol{\Phi} \mathrm{d} V+\int_{\partial \mathcal{B}_{0}} \mathbf{T}_{0}^{\text {ext }} \cdot \delta_{\mathbf{x}} \boldsymbol{\Phi} \mathrm{d} S
$$

and

$$
\mathrm{W}_{\text {conf }}=\int_{\mathcal{B}_{0}} \mathbf{B}_{0}^{d} \cdot \delta_{\mathbf{x}} \mathbf{\Phi} \mathrm{d} V+\int_{\partial \mathcal{B}_{0}} \mathbf{T}_{0}^{d} \cdot \delta_{\mathbf{x}} \mathbf{\Phi} \mathrm{d} S
$$

where $\mathbf{B}_{0}^{\text {ext }}=\mathbf{B}_{0}^{\text {ext }}\left(\mathbf{b}_{0}\right)$ and $\mathbf{B}_{0}^{d}$ are some body forces, $\mathbf{T}_{0}^{\text {ext }}=\mathbf{T}_{0}^{\text {ext }}\left(\mathbf{t}_{0}\right)$ and $\mathbf{T}_{0}^{d}$ are some tractions in reference to $\mathcal{B}_{0}$.

By using the same procedure that is used for (14)-(20), we have the following balance equation and boundary condition of the material motion problem in reference to the material configuration $\mathcal{B}_{0}$ :

$$
\nabla_{\mathbf{X}} \cdot \boldsymbol{\Sigma}+\mathbf{B}_{0}=:-\mathbf{B}_{0}^{d} \quad \text { in } \mathcal{B}_{0} \quad \text { and } \quad \boldsymbol{\Sigma} \cdot \mathbf{N}-\mathbf{T}_{0}^{\text {ext }}=: \mathbf{T}_{0}^{d} \quad \text { on } \partial \mathcal{B}_{0}
$$

where the material motion Cauchy stress tensor $\boldsymbol{\Sigma}$ and the material body force $\mathbf{B}_{0}$ are defined as

$$
\boldsymbol{\Sigma}:=W_{0 \mathbf{f}} \mathbf{I}-\mathbf{F}^{t} \cdot \mathbf{P} \quad \text { and } \quad \mathbf{B}_{0}:=\mathbf{B}_{0}^{i n t}+\mathbf{B}_{0}^{\text {ext }}
$$

in which $\mathbf{B}_{0}^{\text {int }}:=-\partial_{\boldsymbol{\Phi}} W_{0 \mathbf{f}}$. Furthermore, $\mathbf{B}_{0}^{\text {ext }}, \mathbf{B}_{0}^{d}$ can be defined as

$$
\mathbf{B}_{0}^{\text {ext }}:=-\mathbf{f}^{-t} \cdot \mathbf{b}_{0} \quad \text { and } \quad \mathbf{B}_{0}^{d}:=\mathbf{0}
$$


and the definitions for $\mathbf{T}_{0}^{d}, \mathbf{T}_{0}^{\text {ext }}$ are

$$
\mathbf{T}_{0}^{d}:=W_{t \mathbf{f}} \mathbf{N} \quad \text { and } \quad \mathbf{T}_{0}^{e x t}:=-\mathbf{f}^{-t} \cdot \mathbf{t}_{0} .
$$

\section{NONLINEAR ELECTRO-ELASTOSTATICS}

\subsection{Basic Equations in Electrostatics}

In nonlinear electro-elastostatics, we study the deformation of bodies made of hyperelastic material undergoing large deformation and interacting with a static electric field in the absence of magnetic fields, free currents and electric charges. The electric field is governed by Faraday's law:

$$
\nabla_{\mathbf{x}} \times \mathrm{e}=\mathbf{0}
$$

and by electric Gauss' law:

$$
\nabla_{\mathbf{x}} \cdot \mathrm{d}=0
$$

where e is the electric field vector and $d$ is the electric displacement vector in spatial configuration. In material configuration, the Faraday's law (28) and the electric Gauss' law (29) are expressed as

$$
\nabla_{\mathbf{X}} \times \mathrm{E}=\mathbf{0}
$$

and

$$
\nabla_{\mathbf{X}} \cdot \mathrm{D}=0
$$

where $\mathrm{E}$ and $\mathrm{D}$ denote the electric field vector and the electric displacement vector, respectively, in the material configuration $\mathcal{B}_{0}$. The two vectors $\mathrm{E}$ and $\mathrm{D}$ are considered as the pull-back versions of e and $\mathrm{d}$ in $\mathcal{B}_{0}$ :

$$
\mathrm{E}=\mathbf{F}^{t} \cdot \mathrm{e} \quad \text { and } \quad \mathrm{D}=J \mathbf{F}^{-1} \cdot \mathrm{d}
$$

At the boundary of the considered body or across a surface of discontinuity within the body, in the absence of surface charges, the electric field vector and the electric displacement vector must satisfy the jump conditions:

$$
\mathbf{n} \times \llbracket \mathrm{e} \rrbracket=\mathbf{0} \text { and } \llbracket \mathrm{d} \rrbracket \cdot \mathbf{n}=0
$$

where at the boundary: $\llbracket \bullet \rrbracket=\bullet_{\text {outside }}-\bullet_{\text {inside }}$. The counterparts of these jump conditions in the material configuration $\mathcal{B}_{0}$ are written in the form 


$$
\mathbf{N} \times \llbracket \mathrm{E} \rrbracket=\mathbf{0} \quad \text { and } \quad \llbracket \mathrm{D} \rrbracket \cdot \mathbf{N}=0 .
$$

Because the electric field vector e is conservative, this vector can be expressed as the gradient of some scalar electric potential $\psi$ :

$$
\mathrm{e}=-\nabla_{\mathbf{x}} \psi
$$

Similarly, in the material configuration $\mathcal{B}_{0}$, the electric field vector E can be expressed as the gradient of some scalar electric potential $\Psi$ :

$$
\mathrm{E}=-\nabla_{\mathbf{X}} \Psi
$$

where $\Psi$ can be computed as the composition of $\psi$ and $\boldsymbol{\varphi}: \Psi=\psi \circ \boldsymbol{\varphi}$.

The electric displacement $d$ can be computed from the electric field vector e by the relationship

$$
\mathrm{d}=\varepsilon_{0} \mathrm{e}+\mathrm{p}
$$

where $\mathrm{p}$ is the electric polarization density and $\varepsilon_{0}$ is the vacuum electric permittivity. The first term on the right-hand side of (37) is the contribution of free space and the second term is the contribution of condensed matter. In material configuration, the relationship (37) may be cast in the form, [9],

$$
\mathrm{D}=\varepsilon_{0} J \mathbf{C}^{-1} \cdot \mathrm{E}+\mathrm{P}
$$

where $\mathrm{P}=J \mathbf{F}^{-1} \cdot \mathrm{p}$.

The electric body force $\mathbf{b}_{t}^{e}$ that the electric field exerts on matter can be computed by, [34],

$$
\mathbf{b}_{t}^{e}=\left[\nabla_{\mathbf{x}} \mathrm{e}\right] \cdot \mathrm{p} .
$$

By using (28), (29) and (37), this body force can be cast in the form

$$
\mathbf{b}_{t}^{e}=\nabla_{\mathbf{x}} \cdot\left[\mathrm{e} \otimes \mathrm{d}-\frac{1}{2} \varepsilon_{0}[\mathrm{e} \cdot \mathrm{e}] \mathbf{I}\right]
$$

wherein $\otimes$ denotes the dyadic product. In reference to the material configuration $\mathcal{B}_{0}$, this body force can be written as

$$
\mathbf{b}_{0}^{e}=J \mathbf{b}_{t}^{e}=\nabla_{\mathbf{X}}\left[\mathbf{F}^{-t} \cdot \mathrm{E}\right] \cdot \mathrm{P}
$$

or

$$
\mathbf{b}_{0}^{e}=\nabla_{\mathbf{X}} \cdot\left[\mathbf{F}^{-t} \cdot \mathrm{E} \otimes \mathrm{D}-\frac{1}{2} \varepsilon_{0} J\left[\mathrm{E} \cdot \mathbf{C}^{-1} \cdot \mathrm{E}\right] \mathbf{F}^{-t}\right]
$$




\subsection{Spatial Motion Problem}

In nonlinear electro-elastostatics, due to the existence of the electric body force, the Cauchy stress tensor is nonsymmetric. If, by exploiting (40), we define a total stress tensor $\hat{\boldsymbol{\sigma}}$ such that

$$
\hat{\boldsymbol{\sigma}}=\boldsymbol{\sigma}+\mathrm{e} \otimes \mathrm{d}-\frac{1}{2} \varepsilon_{0}[\mathrm{e} \cdot \mathrm{e}] \mathbf{I}
$$

then this total stress tensor is required to be symmetric and the balance equation of linear momentum is written in the form

$$
\nabla_{\mathbf{x}} \cdot \hat{\boldsymbol{\sigma}}+\mathbf{b}_{t}=\mathbf{0}
$$

In reference to the material configuration $\mathcal{B}_{0}$, the counterpart of the balance equation (44) is

$$
\nabla_{\mathbf{X}} \cdot \hat{\mathbf{P}}+\mathbf{b}_{0}=\mathbf{0}
$$

where $\hat{\mathbf{P}}$ is the counterpart of the spatial motion stress tensor $\mathbf{P}$ in nonlinear elastostatics and is considered as the pull-back version of the total stress tensor $\hat{\boldsymbol{\sigma}}$ :

$$
\hat{\mathbf{P}}=J \hat{\boldsymbol{\sigma}} \cdot \mathbf{F}^{-t}
$$

At the boundary of the considered body or across a surface of discontinuity within the body, the jump conditions for $\hat{\boldsymbol{\sigma}}$ and $\hat{\mathbf{P}}$ are

$$
\llbracket \hat{\boldsymbol{\sigma}} \rrbracket \cdot \mathbf{n}=\mathbf{0} \quad \text { and } \quad \llbracket \hat{\mathbf{P}} \rrbracket \cdot \mathbf{N}=\mathbf{0} .
$$

To simplify the problem, let us assume that we only have the jump conditions (33), (34) and (47) at the boundary of the body under consideration. Furthermore, assume that these conditions can be cast in the form $\mathrm{D} \cdot \mathbf{N}=0, \mathrm{~d} \cdot \mathbf{n}=0, \hat{\mathbf{P}} \cdot \mathbf{N}=\hat{\mathbf{t}}_{0}, \hat{\boldsymbol{\sigma}} \cdot \mathbf{n}=\hat{\mathbf{t}}_{t}$. In these conditions, the spatial motion problem in nonlinear electro-elastostatics can be set as the following system of equations:

$$
\begin{aligned}
& \nabla_{\mathbf{X}} \cdot \hat{\mathbf{P}}+\mathbf{b}_{0}=\mathbf{0} \text { and } \nabla_{\mathbf{X}} \cdot \mathrm{D}=0 \quad \text { in } \mathcal{B}_{0} \\
& \hat{\mathbf{P}} \cdot \mathbf{N}=\hat{\mathbf{t}}_{0} \quad \text { and } \quad \mathrm{D} \cdot \mathbf{N}=0 \quad \text { on } \partial \mathcal{B}_{0}
\end{aligned}
$$

in reference to the material configuration $\mathcal{B}_{0}$, or

$$
\begin{aligned}
& \nabla_{\mathbf{x}} \cdot \hat{\boldsymbol{\sigma}}+\mathbf{b}_{t}=\mathbf{0} \quad \text { and } \nabla_{\mathbf{x}} \cdot \mathrm{d}=0 \quad \text { in } \mathcal{B}_{t} \\
& \hat{\boldsymbol{\sigma}} \cdot \mathbf{n}=\hat{\mathbf{t}}_{t} \quad \text { and } \quad \mathrm{d} \cdot \mathbf{n}=0 \quad \text { on } \partial \mathcal{B}_{t}
\end{aligned}
$$

in reference to the spatial configuration $\mathcal{B}_{t}$. 
By assuming the existence of some energy densities that depend on the current state of deformation, on the electric field and on the material placement: $W_{0 \mathrm{e}}(\mathbf{F}, \mathrm{e} ; \mathbf{X}), W_{0 \mathrm{E}}(\mathbf{F}, \mathrm{E} ; \mathbf{X})$, $\left.W_{0 \mathrm{e}}\right|_{\mathbf{F}, \mathrm{e}, \mathbf{X}}=\left.W_{0 \mathrm{EE}}\right|_{\mathbf{F}, \mathrm{E}, \mathbf{X}}$ such that the stress tensor $\boldsymbol{\sigma}$ and the electric polarization density $\mathrm{p}$ can be computed by

$$
\boldsymbol{\sigma}=J^{-1} \mathbf{P} \cdot \mathbf{F}^{t}=J^{-1}\left[\partial_{\mathbf{F}} W_{0 \mathrm{e}}\right] \cdot \mathbf{F}^{t} \text { and } \mathrm{p}=-J^{-1} \partial_{\mathrm{e}} W_{0 \mathrm{e}}
$$

the electric displacements and the total stresses can be shown to be

$$
\mathrm{d}=-J^{-1} \mathbf{F} \cdot \partial_{\mathrm{E}} \hat{W}_{0 \mathbf{F}} ; \quad \hat{\boldsymbol{\sigma}}=J^{-1}\left[\partial_{\mathbf{F}} \hat{W}_{0 \mathbf{F}}\right] \cdot \mathbf{F}^{t}
$$

and

$$
\mathrm{P}=-\partial_{\mathrm{E}} W_{0 \mathrm{E}} ; \quad \mathrm{D}=-\partial_{\mathrm{E}} \hat{W}_{0 \mathbf{F}} ; \quad \hat{\mathbf{P}}=\partial_{\mathbf{F}} \hat{W}_{0 \mathbf{F}}
$$

where the relationships: $\partial_{\mathrm{e}} W_{0 \mathrm{e}}=\mathbf{F} \cdot \partial_{\mathrm{E}} W_{0 \mathrm{E}}$ and $\partial_{\mathbf{F}} W_{0 \mathrm{e}}=\partial_{\mathbf{F}} W_{0 \mathrm{E}}+\left[\mathbf{F}^{-t} \cdot \mathrm{E}\right] \otimes \partial_{\mathrm{E}} W_{0 \mathrm{E}}$, have been used and

$$
\hat{W}_{0 \mathbf{F}}=\hat{W}_{0 \mathbf{F}}(\mathbf{F}, \mathrm{E} ; \mathbf{X})=W_{0 \mathrm{E}}(\mathbf{F}, \mathrm{E} ; \mathbf{X})-\frac{1}{2} \varepsilon_{0} J \mathbf{C}^{-1}:[\mathrm{E} \otimes \mathrm{E}]
$$

in which the double dot denotes the double contraction.

With the relationships (51) and (52) at hand, the condition

$$
\hat{\mathrm{W}}_{i n t}-\hat{\mathrm{W}}_{\text {ext }}=\delta_{\mathbf{X}} \int_{\mathcal{B}_{0}} \hat{W}_{0 \mathbf{F}} \mathrm{d} V-\int_{\mathcal{B}_{0}} \mathbf{b}_{0} \cdot \delta_{\mathbf{X}} \boldsymbol{\varphi} \mathrm{d} V-\int_{\partial \mathcal{B}_{0}} \hat{\mathbf{t}}_{0} \cdot \delta_{\mathbf{X}} \boldsymbol{\varphi} \mathrm{d} S=0
$$

where $\hat{\mathbf{W}}_{\text {int }}$ is the internal and $\hat{\mathrm{W}}_{\text {ext }}$ the external virtual work, with respect to a variation $\delta \mathbf{x}$ of the spatial placement at fixed material placement $\delta \mathbf{X}=\mathbf{0}$, can be shown to be equivalent to the system (48). Furthermore, the system (49) can be shown to be equivalent to the condition

$$
\hat{\mathrm{W}}_{i n t}-\hat{\mathrm{W}}_{\text {ext }}=\delta_{\mathbf{X}} \int_{\mathcal{B}_{t}} \hat{W}_{t \mathbf{F}} \mathrm{d} v-\int_{\mathcal{B}_{t}} \mathbf{b}_{t} \cdot \delta_{\mathbf{X}} \boldsymbol{\varphi} \mathrm{d} v-\int_{\partial \mathcal{B}_{t}} \hat{\mathbf{t}}_{t} \cdot \delta_{\mathbf{X}} \boldsymbol{\varphi} \mathrm{d} s=0 .
$$

\subsection{Material Motion Problem}

In reference to the spatial configuration $\mathcal{B}_{t}$, the balance equations and boundary conditions of the material motion problem in nonlinear electro-elastostatics can be derived by considering the wirtual works with respect to a variation $\delta \mathbf{X}$ of the spatial placement at fixed spatial placement $\delta \mathbf{x}=\mathbf{0}$. Using similar argument as that is used for (10) one obtains

$$
\hat{\mathrm{w}}_{\text {int }}-\hat{\mathrm{w}}_{\text {ext }}-\hat{\mathrm{w}}_{\text {conf }}=0
$$


where the internal virtual work $\hat{\mathrm{w}}_{\text {int }}$ is

$$
\hat{\mathbf{W}}_{i n t}=\delta_{\mathbf{x}} \int_{\mathcal{B}_{t}} \hat{W}_{t \mathbf{f}} \mathrm{d} v=\int_{\mathcal{B}_{t}}\left[\partial_{\mathbf{f}} \hat{W}_{t \mathbf{f}}: \delta_{\mathbf{x}} \mathbf{f}+\partial_{\Phi} \hat{W}_{t \mathbf{f}} \cdot \delta_{\mathbf{x}} \mathbf{\Phi}\right] \mathrm{d} v+\int_{\mathcal{B}_{t}} \partial_{\mathrm{e}} \hat{W}_{t \mathbf{f}} \cdot \delta_{\mathbf{x}} \mathrm{e} \mathrm{d} v .
$$

The external virtual work $\hat{\mathrm{w}}_{\text {ext }}$ can be assumed in the form

$$
\hat{\mathbf{W}}_{e x t}=\int_{\mathcal{B}_{t}} \hat{\mathbf{B}}_{t}^{e x t} \cdot \delta_{\mathbf{x}} \boldsymbol{\Phi} \mathrm{d} v+\int_{\partial \mathcal{B}_{t}} \hat{\mathbf{T}}_{t}^{e x t} \cdot \delta_{\mathbf{x}} \boldsymbol{\Phi} \mathrm{d} s
$$

and $\hat{\mathbf{w}}_{\text {conf }}$ can be written as

$$
\hat{\mathbf{W}}_{\text {conf }}=\int_{\mathcal{B}_{t}} \hat{\mathbf{B}}_{t}^{d} \cdot \delta_{\mathbf{x}} \boldsymbol{\Phi} \mathrm{d} v+\int_{\partial \mathcal{B}_{t}} \hat{\mathbf{T}}_{t}^{d} \cdot \delta_{\mathbf{x}} \boldsymbol{\Phi} \mathrm{d} s .
$$

In the above formulations, $\delta_{\mathbf{x}} \mathrm{e}$ is a variation of the electric field at fixed spatial placement $\delta \mathbf{x}=\mathbf{0}$, the internal potential energy density $\hat{W}_{t \mathbf{f}}$ is defined by: $\hat{W}_{t \mathbf{f}}=\hat{W}_{t \mathbf{f}}(\mathbf{f}, \mathrm{e}, \boldsymbol{\Phi})$, $\left.\hat{W}_{t \mathbf{f}}\right|_{\mathbf{f}, \mathrm{e}, \boldsymbol{\Phi}}=\left.\hat{W}_{t \mathbf{F}}\right|_{\mathbf{F , E}, \mathbf{X}}, \hat{\mathbf{B}}_{t}^{\text {ext }}=\hat{\mathbf{B}}_{t}^{\text {ext }}\left(\mathbf{b}_{t}\right)$ and $\hat{\mathbf{B}}_{t}^{d}$ are some body forces, $\hat{\mathbf{T}}_{t}^{\text {ext }}=\hat{\mathbf{T}}_{t}^{e x t}\left(\hat{\mathbf{t}}_{t}\right)$ and $\hat{\mathbf{T}}_{t}^{d}$ are some tractions. The introduction of (57), (58) and (59) into (56) gives us

$$
\nabla_{\mathbf{x}} \cdot \hat{\mathbf{p}}+\hat{\mathbf{B}}_{t}=:-\hat{\mathbf{B}}_{t}^{d} \quad \text { in } \mathcal{B}_{t} \quad \text { and } \quad \hat{\mathbf{p}} \cdot \mathbf{n}-\hat{\mathbf{T}}_{t}^{e x t}=: \hat{\mathbf{T}}_{t}^{d} \quad \text { on } \partial \mathcal{B}_{t}
$$

where the material motion Piola stress tensor $\hat{\mathbf{p}}$ and the material body force $\hat{\mathbf{B}}_{t}$ are defined as

$$
\hat{\mathbf{p}}:=\partial_{\mathbf{f}} \hat{W}_{t \mathbf{f}} \quad \text { and } \quad \hat{\mathbf{B}}_{t}:=\hat{\mathbf{B}}_{t}^{\text {int }}+\hat{\mathbf{B}}_{t}^{\text {ext }}
$$

wherein $\hat{\mathbf{B}}_{t}^{i n t}:=-\partial_{\boldsymbol{\Phi}} \hat{W}_{t \mathbf{f}}$.

Similar to the case of elastostatics, from the definitions of the stress tensors $\hat{\boldsymbol{\sigma}}$ and $\hat{\mathbf{p}}$, one has the relationships

$$
\hat{\mathbf{p}}=\hat{W}_{t \mathbf{f}} \mathbf{f}^{-t}-\mathbf{f}^{-t} \cdot \hat{\boldsymbol{\sigma}}+\mathbf{f}^{-t} \cdot \mathrm{e} \otimes \mathrm{d} \quad \text { and } \quad \hat{\boldsymbol{\sigma}}=\hat{W}_{t \mathbf{f}} \mathbf{I}-\mathbf{f}^{t} \cdot \hat{\mathbf{p}}+\mathrm{e} \otimes \mathrm{d}
$$

and the balance equation

$$
\nabla_{\mathbf{x}} \cdot \hat{\boldsymbol{\sigma}}-\mathbf{f}^{t} \cdot \hat{\mathbf{B}}_{t}^{\text {ext }}=\mathbf{f}^{t} \cdot \hat{\mathbf{B}}_{t}^{d} \quad \text { in } \mathcal{B}_{t}
$$

which by comparing with the balance equation of linear momentum in (49) gives us the definition

$$
\hat{\mathbf{B}}_{t}^{e x t}:=-\mathbf{f}^{-t} \cdot \mathbf{b}_{t} \quad \text { and } \quad \hat{\mathbf{B}}_{t}^{d}:=\mathbf{0}
$$


With the help of the boundary condition $\hat{\boldsymbol{\sigma}} \cdot \mathbf{n}=\hat{\mathbf{t}}_{t}$ and the relationship (65.1) we can rewrite the boundary condition $(60.2)$ in the form

$$
\hat{W}_{t \mathbf{f}} \mathbf{f}^{-t} \cdot \mathbf{n}-\mathbf{f}^{-t} \cdot \hat{\mathbf{t}}_{t}=\hat{\mathbf{T}}_{t}^{d}+\hat{\mathbf{T}}_{t}^{e x t}
$$

and define $\hat{\mathbf{T}}_{t}^{d}, \hat{\mathbf{T}}_{t}^{e x t}$ as

$$
\hat{\mathbf{T}}_{t}^{d}:=\hat{W}_{t \mathbf{f}} \mathbf{f}^{-t} \cdot \mathbf{n} \quad \text { and } \quad \hat{\mathbf{T}}_{t}^{e x t}:=-\mathbf{f}^{-t} \cdot \hat{\mathbf{t}}_{t}
$$

In reference to the material configuration $\mathcal{B}_{0}$, with respect to a variation $\delta \mathbf{X}$ of the material placement at $\delta \mathbf{x}=\mathbf{0}$, the internal virtual work is

$$
\hat{\mathbf{W}}_{i n t}=\delta_{\mathbf{x}} \int_{\mathcal{B}_{0}} \hat{W}_{0 \mathbf{f}} \mathrm{d} V
$$

where the internal energy density $\hat{W}_{0 \mathbf{f}}$ is defined by $\hat{W}_{0 \mathbf{f}}=\hat{W}_{0 \mathbf{f}}(\mathbf{f}, \mathrm{e}, \boldsymbol{\Phi}),\left.\hat{W}_{0 \mathbf{f}}\right|_{\mathbf{f}, \mathrm{e}, \boldsymbol{\Phi}}=$ $\left.\hat{W}_{0 \mathbf{F}}\right|_{\mathbf{F}, \mathrm{E}, \mathbf{X}}$. Furthermore, we assume

$$
\hat{\mathbf{w}}_{\text {ext }}=\int_{\mathcal{B}_{0}} \hat{\mathbf{B}}_{0}^{e x t} \cdot \delta_{\mathbf{x}} \boldsymbol{\Phi} \mathrm{d} V+\int_{\partial \mathcal{B}_{0}} \hat{\mathbf{T}}_{0}^{e x t} \cdot \delta_{\mathbf{x}} \boldsymbol{\Phi} \mathrm{d} S
$$

and

$$
\hat{\mathbf{W}}_{c o n f}=\int_{\mathcal{B}_{0}} \hat{\mathbf{B}}_{0}^{d} \cdot \delta_{\mathbf{x}} \boldsymbol{\Phi} \mathrm{d} V+\int_{\partial \mathcal{B}_{0}} \hat{\mathbf{T}}_{0}^{d} \cdot \delta_{\mathbf{x}} \boldsymbol{\Phi} \mathrm{d} S
$$

in which $\hat{\mathbf{B}}_{0}^{\text {ext }}=\hat{\mathbf{B}}_{0}^{\text {ext }}\left(\mathbf{b}_{0}\right), \hat{\mathbf{T}}_{0}^{\text {ext }}=\hat{\mathbf{T}}_{0}^{\text {ext }}\left(\hat{\mathbf{t}}_{0}\right), \hat{\mathbf{B}}_{0}^{d}$ and $\hat{\mathbf{T}}_{0}^{d}$ are the counterparts of $\hat{\mathbf{B}}_{t}^{\text {ext }}, \hat{\mathbf{T}}_{t}^{\text {ext }}, \hat{\mathbf{B}}_{t}^{d}$ and $\hat{\mathbf{T}}_{t}^{d}$, respectively.

By introducing (67)-(69) into (56), we have the following balance equation and boundary condition:

$$
\nabla_{\mathbf{X}} \cdot \hat{\boldsymbol{\Sigma}}+\hat{\mathbf{B}}_{0}=:-\hat{\mathbf{B}}_{0}^{d} \quad \text { in } \mathcal{B}_{0} \quad \text { and } \quad \hat{\boldsymbol{\Sigma}} \cdot \mathbf{N}-\hat{\mathbf{T}}_{0}^{e x t}=: \hat{\mathbf{T}}_{0}^{d} \quad \text { on } \partial \mathcal{B}_{0}
$$

where the material motion Cauchy stress tensor $\hat{\boldsymbol{\Sigma}}$ and the material body force $\hat{\mathbf{B}}_{0}$ are defined as

$$
\hat{\boldsymbol{\Sigma}}:=\hat{W}_{0 \mathbf{f}} \mathbf{I}-\mathbf{F}^{t} \cdot \hat{\mathbf{P}}+\mathrm{E} \otimes \mathrm{D} \quad \text { and } \quad \hat{\mathbf{B}}_{0}:=\hat{\mathbf{B}}_{0}^{\text {int }}+\hat{\mathbf{B}}_{0}^{\text {ext }}
$$

in which $\hat{\mathbf{B}}_{0}^{i n t}:=-\partial_{\Phi} \hat{W}_{0 \mathbf{f}}$. Besides, the definition for $\hat{\mathbf{B}}_{0}^{\text {ext }}, \hat{\mathbf{B}}_{0}^{d}$ is

$$
\hat{\mathbf{B}}_{0}^{\text {ext }}:=-\mathbf{f}^{-t} \cdot \mathbf{b}_{0} \quad \text { and } \quad \hat{\mathbf{B}}_{0}^{d}:=\mathbf{0}
$$


and for $\hat{\mathbf{T}}_{0}^{d}, \hat{\mathbf{T}}_{0}^{e x t}$,

$$
\hat{\mathbf{T}}_{0}^{d}:=\hat{W}_{t \mathbf{f}} \mathbf{N} \quad \text { and } \quad \hat{\mathbf{T}}_{0}^{e x t}:=-\mathbf{f}^{-t} \cdot \hat{\mathbf{t}}_{0}
$$

\section{NONLINEAR MAGNETO-ELASTOSTATICS}

\subsection{Basic Equations in Magnetostatics}

In nonlinear magneto-elastostatics, we study the deformation of bodies made of hyperelastic materials undergoing large deformation and interacting with a static magnetic field. In the absence of electric current, the magnetic field is governed by Ampere's law:

$$
\nabla_{\mathbf{x}} \times \mathrm{h}=\mathbf{0}
$$

and by Gauss's law for magnetism:

$$
\nabla_{\mathbf{x}} \cdot \mathrm{b}=0
$$

where $\mathrm{h}$ and $\mathrm{b}$ denote respectively the magnetic field vector and the magnetic induction vector in the spatial configuration $\mathcal{B}_{t}$. In reference to the material configuration $\mathcal{B}_{0}$, these laws have the form

$$
\nabla_{\mathbf{X}} \times \mathrm{H}=\mathbf{0}
$$

and

$$
\nabla_{\mathbf{X}} \cdot \mathrm{B}=0
$$

where $\mathrm{H}$ is the magnetic field vector and $\mathrm{B}$ is the magnetic induction vector in material configuration. $\mathrm{H}$ and $\mathrm{B}$ are considered as the pull-back versions of $\mathrm{h}$ and $\mathrm{b}$ in $\mathcal{B}_{0}$ :

$$
\mathrm{H}=\mathbf{F}^{t} \cdot \mathrm{h} \quad \text { and } \quad \mathrm{B}=J \mathbf{F}^{-1} \cdot \mathrm{b} .
$$

In the absence of surface currents, the magnetic field vector and the magnetic induction vector must satisfy the jump conditions

$$
\mathbf{n} \times \llbracket \mathrm{h} \rrbracket=\mathbf{0} \text { and } \mathbf{n} \cdot \llbracket \mathrm{b} \rrbracket=\mathbf{0}
$$

in reference to the deformed configuration $\mathcal{B}_{t}$, or

$$
\mathbf{N} \times \llbracket \mathrm{H} \rrbracket=\mathbf{0} \text { and } \mathbf{N} \cdot \llbracket \mathrm{B} \rrbracket=\mathbf{0}
$$

in reference to the undeformed configuration $\mathcal{B}_{0}$. 
Because the magnetic induction is divergence free, in reference to the deformed configuration $\mathcal{B}_{t}$, a magnetic vector potential a is defined such that

$$
\mathrm{b}=\nabla_{\mathrm{x}} \times \mathrm{a}
$$

which has its counterpart in $\mathcal{B}_{0}$ as

$$
\mathrm{B}=\nabla_{\mathbf{x}} \times \mathrm{A}
$$

where $\mathrm{A}=\mathbf{F}^{t} \cdot \mathrm{a}$.

The relationship between the magnetic induction $\mathrm{b}$ and the magnetic field $\mathrm{h}$ can be written as

$$
\mathrm{b}=\mu_{0} \mathrm{~h}+\mu_{0} \mathrm{~m}
$$

where $\mathrm{m}$ is the magnetization vector and $\mu_{0}$ is the vacuum magnetic permittivity. The first term on the right-hand side of (83) is the contribution of free space and the second term is the contribution of condensed matter. In material configuration, the relationship (83) between the magnetic induction and the magnetic field has the form

$$
J^{-1} \mathbf{C} \cdot \mathbf{B}=\mu_{0} \mathrm{H}+\mu_{0} \mathbf{M}
$$

where $\mathrm{M}$ is the magnetization vector in material configuration:

$$
\mathrm{M}=\mathbf{F}^{t} \cdot \mathrm{m} .
$$

The magnetic field exerts on matter a body force $\mathbf{b}_{t}^{m}$, which can be computed as, ([34]),

$$
\mathbf{b}_{t}^{m}=\left[\nabla_{\mathbf{x}} \mathrm{b}\right]^{t} \cdot \mathrm{m}
$$

or by using (74), (75) and (83),

$$
\mathbf{b}_{t}^{m}=\nabla_{\mathbf{x}} \cdot\left[\mu_{0}^{-1}\left[\mathrm{~b} \otimes \mathrm{b}-\frac{1}{2}[\mathrm{~b} \cdot \mathrm{b}] \mathbf{I}\right]+[\mathrm{m} \cdot \mathrm{b}] \mathbf{I}-\mathrm{m} \otimes \mathbf{b}\right] .
$$

In reference to the material configuration $\mathcal{B}_{0}$, this body force can be computed as

$$
\mathbf{b}_{0}^{m}=J \mathbf{b}_{t}^{m}=J \mathbf{F}^{-t} \cdot\left[\nabla_{\mathbf{X}}\left[J^{-1} \mathbf{F} \cdot \mathrm{B}\right]\right]^{t} \cdot \mathbf{F}^{-t} \cdot \mathrm{M}
$$

or equivalently

$$
\mathbf{b}_{0}^{m}=\nabla_{\mathbf{X}} \cdot\left[\mu_{0}^{-1} J^{-1}\left[\mathbf{F} \cdot \mathrm{B} \otimes \mathrm{B}-\frac{1}{2}[\mathrm{~B} \cdot \mathbf{C} \cdot \mathrm{B}] \mathbf{F}^{-t}\right]+[\mathrm{B} \cdot \mathrm{M}] \mathbf{F}^{-t}-\mathbf{F}^{-t} \cdot \mathrm{M} \otimes \mathrm{B}\right] .
$$




\subsection{Spatial Motion Problem}

Similar to nonlinear electro-elastostatics, in nonlinear magneto-elastostatics due to the existence of the magnetic body force, the Cauchy stress tensor is nonsymmetric. If, by employing (87), we define a total stress tensor $\tilde{\boldsymbol{\sigma}}$ such that

$$
\tilde{\boldsymbol{\sigma}}=\boldsymbol{\sigma}+\left[\mu_{0}^{-1}\left[\mathrm{~b} \otimes \mathrm{b}-\frac{1}{2}[\mathrm{~b} \cdot \mathrm{b}] \mathbf{I}\right]+[\mathrm{m} \cdot \mathrm{b}] \mathbf{I}-\mathrm{m} \otimes \mathrm{b}\right]
$$

then the balance equation of linear momentum of the nonlinear magneto-elastostatic problem becomes

$$
\nabla_{\mathbf{x}} \cdot \tilde{\boldsymbol{\sigma}}+\mathbf{b}_{t}=\mathbf{0}
$$

In reference to the material configuration, the counterpart of (91) reads

$$
\nabla_{\mathbf{X}} \cdot \tilde{\mathbf{P}}+\mathbf{b}_{0}=\mathbf{0}
$$

where $\tilde{\mathbf{P}}$ is the counterpart of the spatial motion stress tensor $\mathbf{P}$ in nonlinear elastostatics. This stress tensor can be considered as the pull-back version of the total stress tensor $\tilde{\boldsymbol{\sigma}}$ in material configuration:

$$
\tilde{\mathbf{P}}=J \tilde{\boldsymbol{\sigma}} \cdot \mathbf{F}^{-t}
$$

At the boundary of the considered body or across a surface of discontinuity within the body, the jump conditions for $\tilde{\boldsymbol{\sigma}}$ and $\tilde{\mathbf{P}}$ are

$$
\llbracket \hat{\boldsymbol{\sigma}} \rrbracket \cdot \mathbf{n}=\mathbf{0} \text { and } \llbracket \tilde{\mathbf{P}} \rrbracket \cdot \mathbf{N}=\mathbf{0}
$$

which take into account both magnetic and mechanical contributions.

For the sake of simplicity, let us assume that we only have the jump conditions (79), (80) and (94) at the boundary of the body under consideration such that: $\mathbf{N} \times \mathrm{H}=\mathbf{0}$, $\mathbf{n} \times \mathrm{h}=\mathbf{0}, \tilde{\mathbf{P}} \cdot \mathbf{N}=\tilde{\mathbf{t}}_{0}, \tilde{\boldsymbol{\sigma}} \cdot \mathbf{n}=\tilde{\mathbf{t}}_{t}$. In this case, the spatial motion problem in nonlinear magneto-elastostatics can be set as

$$
\begin{aligned}
& \nabla_{\mathbf{X}} \cdot \tilde{\mathbf{P}}+\mathbf{b}_{0}=\mathbf{0} \text { and } \nabla_{\mathbf{X}} \times \mathrm{H}=\mathbf{0} \quad \text { in } \mathcal{B}_{0} \\
& \tilde{\mathbf{P}} \cdot \mathbf{N}=\tilde{\mathbf{t}}_{0} \quad \text { and } \quad \mathbf{N} \times \mathrm{H}=\mathbf{0} \quad \text { on } \partial \mathcal{B}_{0}
\end{aligned}
$$

in reference to the material configuration $\mathcal{B}_{0}$, or

$$
\begin{aligned}
& \nabla_{\mathbf{x}} \cdot \tilde{\boldsymbol{\sigma}}+\mathbf{b}_{t}=\mathbf{0} \text { and } \nabla_{\mathbf{x}} \times \mathrm{h}=\mathbf{0} \quad \text { in } \mathcal{B}_{t} \\
& \tilde{\boldsymbol{\sigma}} \cdot \mathbf{n}=\tilde{\mathbf{t}}_{t} \text { and } \quad \mathbf{n} \times \mathbf{h}=\mathbf{0} \quad \text { on } \partial \mathcal{B}_{t}
\end{aligned}
$$

in reference to the spatial configuration $\mathcal{B}_{t}$. 
By assuming the existence of some energy densities that depend on the current state of deformation, on the magnetic induction and on the material placement $W_{0 b}(\mathbf{F}, \mathbf{b} ; \mathbf{X})$, $W_{0 B}(\mathbf{F}, \mathbf{B} ; \mathbf{X}),\left.W_{0 b}\right|_{\mathbf{F}, \mathrm{b}, \mathbf{X}}=\left.W_{0 B}\right|_{\mathbf{F}, \mathrm{B}, \mathbf{X}}$, such that the stress tensor $\boldsymbol{\sigma}$ and the magnetization vector $\mathrm{m}$ can be computed by

$$
\boldsymbol{\sigma}=J^{-1}\left[\partial_{\mathbf{F}} W_{0 b}\right] \cdot \mathbf{F}^{t} \text { and } \mathrm{m}=-J^{-1} \partial_{\mathrm{b}} W_{0 b}
$$

one obtains the following formulations for $\mathrm{h}, \tilde{\boldsymbol{\sigma}}$ :

$$
\mathrm{h}=\mathbf{F}^{-t} \cdot \partial_{\mathrm{B}} \tilde{W}_{0 \mathbf{F}} ; \quad \hat{\boldsymbol{\sigma}}=J^{-1}\left[\partial_{\mathbf{F}} \tilde{W}_{0 \mathbf{F}}\right] \cdot \mathbf{F}^{t}
$$

and for $\mathrm{M}, \mathrm{H}, \tilde{\mathbf{P}}$ :

$$
\mathbf{M}=-\partial_{\mathrm{B}} W_{0 B} ; \quad \mathrm{H}=\partial_{\mathrm{B}} \tilde{W}_{0 \mathbf{F}} ; \quad \tilde{\mathbf{P}}=\partial_{\mathbf{F}} \tilde{W}_{0 \mathbf{F}}
$$

in which the energy density $\tilde{W}_{0 \mathbf{F}}$ is defined as

$$
\tilde{W}_{0 \mathbf{F}}=\tilde{W}_{0 \mathbf{F}}(\mathbf{F}, \mathbf{B} ; \mathbf{X})=W_{0 \mathrm{~B}}(\mathbf{F}, \mathbf{B} ; \mathbf{X})+\frac{1}{2} \mu_{0}^{-1} J^{-1} \mathbf{C}:[\mathrm{B} \otimes \mathrm{B}] .
$$

By using (97)-(100), it can be shown that the system (95) is equivalent to the condition

$$
\tilde{\mathbf{W}}_{i n t}-\tilde{\mathbf{W}}_{\text {ext }}=\delta_{\mathbf{X}} \int_{\mathcal{B}_{0}} \tilde{W}_{0 \mathbf{F}} \mathrm{d} V-\int_{\mathcal{B}_{0}} \mathbf{b}_{0} \cdot \delta_{\mathbf{X}} \boldsymbol{\varphi} \mathrm{d} V-\int_{\partial \mathcal{B}_{0}} \tilde{\mathbf{t}}_{0} \cdot \delta_{\mathbf{X}} \boldsymbol{\varphi} \mathrm{d} S=0
$$

where $\tilde{W}_{i n t}$ and $\tilde{W}_{\text {ext }}$ are the internal and external virtual work, respectively, with respect to a variation $\delta \mathbf{x}$ of the spatial placement at fixed material placement $\delta \mathbf{X}=\mathbf{0}$.

Similarly, the system (96) is equivalent to the condition

$$
\tilde{\mathbf{W}}_{i n t}-\tilde{\mathrm{W}}_{\text {ext }}=\delta_{\mathbf{X}} \int_{\mathcal{B}_{t}} \tilde{W}_{t \mathbf{F}} \mathrm{d} v-\int_{\mathcal{B}_{t}} \mathbf{b}_{t} \cdot \delta_{\mathbf{X}} \boldsymbol{\varphi} \mathrm{d} v-\int_{\partial \mathcal{B}_{t}} \tilde{\mathbf{t}}_{t} \cdot \delta_{\mathbf{X}} \boldsymbol{\varphi} \mathrm{d} s=0
$$

where the internal energy density $\tilde{W}_{t \mathbf{F}}$ is defined as $\tilde{W}_{t \mathbf{F}}=\tilde{W}_{t \mathbf{F}}(\mathbf{F}, \mathrm{B} ; \mathbf{X}),\left.\quad \tilde{W}_{t \mathbf{F}}\right|_{\mathbf{F}, \mathrm{B}, \mathbf{X}}=$ $\left.J^{-1} \tilde{W}_{0 \mathbf{F}}\right|_{\mathbf{F}, \mathrm{B}, \mathbf{X}}$.

\subsection{Material Motion Problem}

By using a similar procedure that is used above for nonlinear elastostatics and nonlinear electro-elastostatics, in reference to the spatial configuration $\mathcal{B}_{t}$, the balance equations and boundary conditions of the material motion problem in nonlinear magneto-elastostatics can be derived by considering the virtual works with respect to a variation $\delta \mathbf{X}$ of the spatial placement at fixed spatial placement $\delta \mathbf{x}=\mathbf{0}$ : 


$$
\tilde{\mathbf{W}}_{i n t}-\tilde{\mathbf{W}}_{\text {ext }}-\tilde{\mathrm{w}}_{\text {conf }}=0
$$

where the internal virtual work $\tilde{\mathrm{w}}_{\text {int }}$ is

$$
\tilde{\mathbf{W}}_{i n t}=\delta_{\mathbf{x}} \int_{\mathcal{B}_{t}} \tilde{W}_{t \mathbf{f}} \mathrm{d} v=\int_{\mathcal{B}_{t}}\left[\partial_{\mathbf{f}} \tilde{W}_{t \mathbf{f}}: \delta_{\mathbf{x}} \mathbf{f}+\partial_{\Phi} \tilde{W}_{t \mathbf{f}} \cdot \delta_{\mathbf{x}} \mathbf{\Phi}\right] \mathrm{d} v+\int_{\mathcal{B}_{t}} \partial_{\mathrm{b}} \tilde{W}_{t \mathbf{f}} \cdot \delta_{\mathbf{x}} \mathrm{b} \mathrm{d} v
$$

the external virtual work $\tilde{\mathbf{W}}_{\text {ext }}$ can be assumed in the form

$$
\tilde{\mathbf{W}}_{\text {ext }}=\int_{\mathcal{B}_{t}} \tilde{\mathbf{B}}_{t}^{\text {ext }} \cdot \delta_{\mathbf{x}} \boldsymbol{\Phi} \mathrm{d} v+\int_{\partial \mathcal{B}_{t}} \tilde{\mathbf{T}}_{t}^{e x t} \cdot \delta_{\mathbf{x}} \boldsymbol{\Phi} \mathrm{d} s
$$

and $\tilde{\mathbf{w}}_{\text {conf }}$ can be written as

$$
\tilde{\mathbf{W}}_{\text {conf }}=\int_{\mathcal{B}_{t}} \tilde{\mathbf{B}}_{t}^{d} \cdot \delta_{\mathbf{x}} \boldsymbol{\Phi} \mathrm{d} v+\int_{\partial \mathcal{B}_{t}} \tilde{\mathbf{T}}_{t}^{d} \cdot \delta_{\mathbf{x}} \boldsymbol{\Phi} \mathrm{d} s
$$

In the above formulations, $\delta_{\mathbf{x}} \mathrm{b}$ is a variation of the magnetic induction at fixed spatial placement $\delta \mathbf{x}=\mathbf{0}$, the internal potential energy density $\tilde{W}_{t \mathbf{f}}$ is defined by $\tilde{W}_{t \mathbf{f}}=\tilde{W}_{t \mathbf{f}}(\mathbf{f}, \mathrm{b}, \mathbf{\Phi})$, $\left.\tilde{W}_{t \mathbf{f}}\right|_{\mathbf{f}, \mathrm{b}, \boldsymbol{\Phi}}=\left.\tilde{W}_{t \mathbf{F}}\right|_{\mathbf{F}, \mathbf{B}, \mathbf{X}}, \tilde{\mathbf{B}}_{t}^{\text {ext }}=\tilde{\mathbf{B}}_{t}^{\text {ext }}\left(\mathbf{b}_{t}\right), \tilde{\mathbf{T}}_{t}^{e x t}=\tilde{\mathbf{T}}_{t}^{\text {ext }}\left(\tilde{\mathbf{t}}_{t}\right), \tilde{\mathbf{B}}_{t}^{d}$ and $\tilde{\mathbf{T}}_{t}^{d}$ are the counterparts of $\hat{\mathbf{B}}_{t}^{\text {ext }}, \hat{\mathbf{T}}_{t}^{\text {ext }}, \hat{\mathbf{B}}_{t}^{d}$ and $\hat{\mathbf{T}}_{t}^{d}$, respectively.

With respect to (104), (105) and (106), the corresponding balance equation and boundary condition reads

$$
\nabla_{\mathbf{x}} \cdot \tilde{\mathbf{p}}+\tilde{\mathbf{B}}_{t}=:-\tilde{\mathbf{B}}_{t}^{d} \quad \text { in } \mathcal{B}_{t} \quad \text { and } \quad \tilde{\mathbf{p}} \cdot \mathbf{n}-\tilde{\mathbf{T}}_{t}^{e x t}=: \tilde{\mathbf{T}}_{t}^{d} \quad \text { on } \partial \mathcal{B}_{t}
$$

where the material motion Piola stress tensor $\tilde{\mathbf{p}}$ and the material body force $\tilde{\mathbf{B}}_{t}$ are defined as

$$
\tilde{\mathbf{p}}:=\partial_{\mathbf{f}} \tilde{W}_{t \mathbf{f}} \quad \text { and } \quad \tilde{\mathbf{B}}_{t}:=\tilde{\mathbf{B}}_{t}^{\text {int }}+\tilde{\mathbf{B}}_{t}^{\text {ext }}
$$

in which $\tilde{\mathbf{B}}_{t}^{i n t}:=-\partial_{\boldsymbol{\Phi}} \tilde{W}_{t \mathbf{f}}$. From these definitions we have the relationships:

$$
\begin{aligned}
\tilde{\mathbf{p}} & =\tilde{W}_{t \mathbf{f}} \mathbf{f}^{-t}-\mathbf{f}^{-t} \cdot \tilde{\boldsymbol{\sigma}}-[\mathrm{b} \cdot \mathrm{h}] \mathbf{f}^{-t}+\mathbf{f}^{-t} \cdot \mathrm{h} \otimes \mathrm{b} \\
\text { and } \quad \tilde{\boldsymbol{\sigma}} & =\tilde{W}_{t \mathbf{f}} \mathbf{I}-\mathbf{f}^{t} \cdot \tilde{\mathbf{p}}-[\mathrm{b} \cdot \mathrm{h}] \mathbf{I}+\mathrm{h} \otimes \mathrm{b}
\end{aligned}
$$

the definition of $\tilde{\mathbf{B}}_{t}^{\text {ext }}, \tilde{\mathbf{B}}_{t}^{d}$ :

$$
\tilde{\mathbf{B}}_{t}^{e x t}:=-\mathbf{f}^{-t} \cdot \mathbf{b}_{t} \quad \text { and } \quad \tilde{\mathbf{B}}_{t}^{d}:=\mathbf{0}
$$

and the definition of $\tilde{\mathbf{T}}_{t}^{d}, \tilde{\mathbf{T}}_{t}^{\text {ext }}$ : 


$$
\tilde{\mathbf{T}}_{t}^{d}:=\tilde{W}_{t f} \mathbf{f}^{-t} \cdot \mathbf{n} \quad \text { and } \quad \tilde{\mathbf{T}}_{t}^{e x t}:=-\mathbf{f}^{-t} \cdot \tilde{\mathbf{t}}_{t}
$$

In reference to the material configuration $\mathcal{B}_{0}$, with respect to a variation $\delta \mathbf{X}$ of the material placement at $\delta \mathbf{x}=\mathbf{0}$, the internal virtual work $\tilde{\mathrm{w}}_{i n t}$ is

$$
\tilde{\mathrm{W}}_{i n t}=\delta_{\mathbf{x}} \int_{\mathcal{B}_{0}} \tilde{W}_{0 \mathbf{f}} \mathrm{d} V
$$

the external virtual work $\tilde{\mathrm{w}}_{\text {ext }}$ can be assumed as

$$
\tilde{\mathbf{W}}_{\text {ext }}=\int_{\mathcal{B}_{0}} \tilde{\mathbf{B}}_{0}^{e x t} \cdot \delta_{\mathbf{x}} \boldsymbol{\Phi} \mathrm{d} V+\int_{\partial \mathcal{B}_{0}} \tilde{\mathbf{T}}_{0}^{e x t} \cdot \delta_{\mathbf{x}} \boldsymbol{\Phi} \mathrm{d} S
$$

and $\tilde{\mathbf{w}}_{\text {conf }}$ can be written as

$$
\tilde{\mathbf{W}}_{\text {conf }}=\int_{\mathcal{B}_{0}} \tilde{\mathbf{B}}_{0}^{d} \cdot \delta_{\mathbf{x}} \boldsymbol{\Phi} \mathrm{d} V+\int_{\partial \mathcal{B}_{0}} \tilde{\mathbf{T}}_{0}^{d} \cdot \delta_{\mathbf{x}} \boldsymbol{\Phi} \mathrm{d} S
$$

where the internal energy density $\tilde{W}_{0 \mathbf{f}}$ is defined by $\tilde{W}_{0 \mathbf{f}}=\tilde{W}_{0 \mathbf{f}}(\mathbf{f}, \mathrm{b}, \boldsymbol{\Phi}),\left.\quad \tilde{W}_{0 \mathbf{f}}\right|_{\mathbf{f}, \mathrm{b}, \boldsymbol{\Phi}}=$ $\left.\tilde{W}_{0 \mathbf{F}}\right|_{\mathbf{F}, \mathbf{B}, \mathbf{X}}, \tilde{\mathbf{B}}_{0}^{\text {ext }}=\tilde{\mathbf{B}}_{0}^{\text {ext }}\left(\mathbf{b}_{0}\right), \tilde{\mathbf{T}}_{0}^{\text {ext }}=\tilde{\mathbf{T}}_{0}^{\text {ext }}\left(\tilde{\mathbf{t}}_{0}\right), \tilde{\mathbf{B}}_{0}^{d}$ and $\tilde{\mathbf{T}}_{0}^{d}$ are the counterparts of $\tilde{\mathbf{B}}_{t}^{\text {ext }}, \tilde{\mathbf{T}}_{t}^{\text {ext }}, \tilde{\mathbf{B}}_{t}^{d}$ and $\tilde{\mathbf{T}}_{t}^{d}$, respectively.

Corresponding to (112), (113) and (114), we have the following balance equation and boundary condition:

$$
\nabla_{\mathbf{X}} \cdot \tilde{\boldsymbol{\Sigma}}+\tilde{\mathbf{B}}_{0}=:-\tilde{\mathbf{B}}_{0}^{d} \quad \text { in } \mathcal{B}_{0} \quad \text { and } \quad \tilde{\boldsymbol{\Sigma}} \cdot \mathbf{N}-\tilde{\mathbf{T}}_{0}^{e x t}=: \tilde{\mathbf{T}}_{0}^{d} \quad \text { on } \partial \mathcal{B}_{0}
$$

where the material motion Cauchy stress tensor $\tilde{\boldsymbol{\Sigma}}$ and the material body force $\tilde{\mathbf{B}}_{0}$ are defined as

$$
\tilde{\boldsymbol{\Sigma}}=\tilde{W}_{0 \mathbf{f}} \mathbf{I}-\mathbf{F}^{t} \cdot \tilde{\mathbf{P}}-[\mathrm{B} \cdot \mathrm{H}] \mathbf{I}+\mathrm{H} \otimes \mathrm{B} \quad \text { and } \quad \tilde{\mathbf{B}}_{0}:=\tilde{\mathbf{B}}_{0}^{i n t}+\tilde{\mathbf{B}}_{0}^{\text {ext }}
$$

in which $\tilde{\mathbf{B}}_{0}^{\text {int }}:=-\partial_{\Phi} \tilde{W}_{0 \mathrm{f}}$. In addition, the definition for $\tilde{\mathbf{B}}_{0}^{\text {ext }}, \tilde{\mathbf{B}}_{0}^{d}$ is

$$
\tilde{\mathbf{B}}_{0}^{e x t}:=-\mathbf{f}^{-t} \cdot \mathbf{b}_{0} \quad \text { and } \quad \tilde{\mathbf{B}}_{0}^{d}:=\mathbf{0}
$$

and for $\tilde{\mathbf{T}}_{0}^{d}, \tilde{\mathbf{T}}_{0}^{\text {ext }}$ :

$$
\tilde{\mathbf{T}}_{0}^{d}:=\tilde{W}_{0 \mathbf{f}} \mathbf{N} \quad \text { and } \quad \tilde{\mathbf{T}}_{0}^{e x t}:=-\mathbf{f}^{-t} \cdot \tilde{\mathbf{t}}_{0}
$$




\section{CONCLUSION}

In this work, material and spatial motion problems in nonlinear electro- and magnetoelastostatics are considered without the assumption that mechanical loads can be derived explicitly from some potential. The corresponding variational formulations and governing equations are derived by a virtual work approach and have very similar forms, which can be represented by

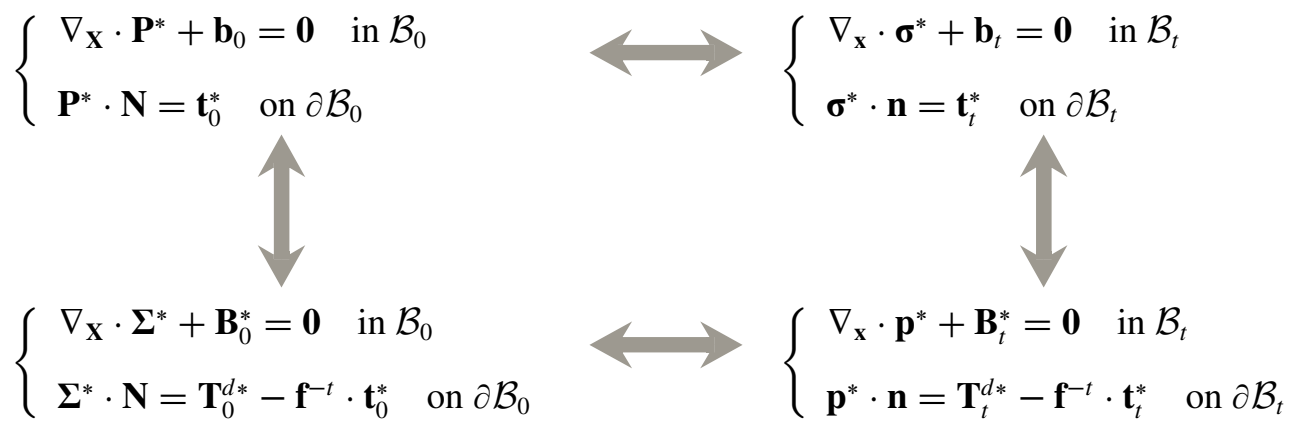

where $(\bullet)^{*}=\{(\bullet),(\hat{\bullet}),(\tilde{\bullet})\}$.

Due to their similarity, the three problems of elastostatics, electro-elastostatics and magneto-elastostatics can be treated in a similar way. Further studies are needed in order to extend the current result to more general boundary conditions for the electric and magnetic fields.

Acknowledgments. The authors gratefully acknowledge financial support of the German Research Foundation (DFG) under the project "Electronic electroactive polymers under electric loading: Experiment, Modelling and Simulation", Grant No. STE 544/36-1 and the project "On the Modelling and Computation of Magneto-Sensitive-Elastomers", Grant No. STE 544/32-1.

\section{REFERENCES}

[1] Bar-Cohen, Y. Electro-active polymers: current capabilities and challenges, in Proceedings of EAPAD Conference (San Diego, CA, March 18-21), pp. 4695-4702, 2002.

[2] Carlson, J. D. and Jolly, M. R. MR fluid, foam and elastomer devices. Mechatronics, 10, 555-569 (2000).

[3] Kordonsky, W. Magneto-rheological effects as a base of new devices and technologies. Journal of Magnetism and Magnetic Materials, 122, 395-398 (1993).

[4] Brigdanov, I. A., Dorfmann, A. Mathematical modeling of magneto-sensitive elastomers. International Journal of Solids and Structures, 40, 4659-4674 (2003).

[5] Bustamante, R., Dorfmann, A. and Ogden R. W. A nonlinear magnetoelastic tube under extension and inflation in an axial magnetic field: numerical solution. Journal of Engineering Mathematics, 59, 139-153 (2007).

[6] Bustamante, R., Dorfmann, A. and Ogden R. W. On variational formulations in nonlinear magnetoelastostatics. Mathematics and Mechanics of Solids, 13, 725-745 (2008).

[7] Dorfmann, A. and Ogden, R. W. Magnetoelastic modelling of elastomers. European Journal of Mechanics ASolids, 22, 497-507 (2003).

[8] Dorfmann, A. and Ogden, R. W. Nonlinear magnetoelastic deformations of elastomers. Acta Mechanica, 167, 13-28 (2004).

[9] Dorfmann, A. and Ogden, R. W. Nonlinear electroelasticity. Acta Mechanica, 174, 167-183 (2005). 
[10] Goulbourne, N., Frecker, M., Mockensturm, E. and Snyder, A. Modeling of a dielectric elastomer diaphragm for a prosthetic blood pump. Proceedings of SPIE-Smart Structures and Materials: Electroactive Polymer Actuators and Devices, 5051, 319-331 (2003)

[11] Jolly, M. R., Carlson, J. D. and Munoz, B. C. A model of the behavior of magneto-rheological materials. Smart Materials and Structures, 5, 607-614 (1996).

[12] Kim, H., Oh, S., Hwang, K., Choi, H., Jeon, J. W. and Nam, J. D. Actuator model of electrostrictive polymers (EPs) for microactuators. Proceedings of SPIE-Smart Structures and Materials: Electroactive Polymer Actuators and Devices, 4329, 482-490 (2001).

[13] Kofod, G. Dielectric elastomer actuators, Ph.D. Thesis, Riso-R-1286 (EN), Denmark, 2001.

[14] Kofod, G., Sommer-Larsen, P., Kornbluh, R. and Pelrine, R. Actuation response of polyacrylate dielectric elastomers. Proceedings of SPIE_Smart Structures and Materials: Electroactive Polymer Actuators and Devices, 4329, 141-147 (2001).

[15] Mockensturm, E. and Goulbourne, N. Dynamic response of dielectric elastomers. International Journal of Nonlinear Mechanics, 41, 388-395 (2006).

[16] Voltairas, P. A., Fotiadis, D. I. and Massalas, C. V. A theoretical study of the hyperelasticity of electro-gels. Proceedings of the Royal Society A, 459, 2121-2130 (2003).

[17] Vu, D. K., Steinmann, P. and Possart, G. Numerical modeling of nonlinear electroelasticity. International Journal of Numerical Methods in Engineering, 70, 685-704 (2006).

[18] Wissler, M. and Mazza, E. Modeling of a pre-strained circular actuator made of dielectric elastomers. Sensors and Actuators A, 120, 184-192 (2005).

[19] Wissler, M. and Mazza, E. Modeling and simulation of dielectric elastomer actuators. Smart Materials and Structures, 14, 1396-1402 (2005).

[20] Yang, E., Frecker, M. and Mockensturm, E. Viscoelastic model of dielectric elastomer membranes. Proceedings of SPIE-Smart Structures and Materials: Electroactive Polymer Actuators and Devices, 5759, 82-93 (2005).

[21] Yang, E., Frecker, M. and Mockensturm, E. Finite element and experimental analyses of non-axisymmetric dielectric elastomer actuators. Proceedings of SPIE_Smart Structures and Materials: Electroactive Polymer Actuators and Devices, 6168, 127-135 (2006).

[22] Yang, E., Frecker, M., Mockensturm, E. and Wu, D. Analytical model and experimental characterization of a dielectric elastomer annulus actuator undergoing large quasi-static deformation. Proceedings of SPIE-Smart Structures and Materials: Smart Structures and Integrated Systems, 5390, 183-193 (2004).

[23] Epstein, M., and Maugin, G. A. Energy-momentum tensor and J-integral in electrodeformable bodies. International Journal of Applied Electromagnetics and Materials, 2, 141-145 (1991).

[24] Huang, Y. N. and Batra, R. C. Energy-momentum tensors in nonsimple elastic dielectrics. Journal of Elasticity, 42, 275-281 (1996).

[25] Kalpakides, V. K. and Agiasofitou, E. K. On material equations in second gradient electroelasticity. Journal of Elasticity, 67, 205-227 (2002).

[26] Maugin, G. A. Material Inhomogeneities in Elasticity, Chapman and Hall, 1993.

[27] Maugin, G. A. and Epstein, M. The electroelastic energy-momentum tensor. Proceedings of the Royal Society of London A, 433, 299-312 (1991).

[28] Pak, Y. E. and Herrmann, G. Conservation laws and the material momentum tensor for the elastic dielectric. International Journal of Engineering Science, 24, 1365-1374 (1986).

[29] Pak, Y. E. and Herrmann, G. Crack extension force in a dielectric medium. International Journal of Engineering Science, 24, 1375-1388 (1986).

[30] Trimarco, C. Material electromagnetic fields and material forces. Archives of Applied Mechanics, 77, 177-184 (2007).

[31] Yavari, A., Marsden, J. E. and Ortiz, M. On Spatial and Material Covariant Balance Laws in Elasticity. Journal of Mathematical Physics, 47, 1-53 (2006).

[32] Vu, D. K. and Steinmann P. Nonlinear electro- and magneto-elastostatics: Material and spatial settings. International Journal of Solids and Structures, 44, 7891-7905 (2007).

[33] Steinmann, P. On boundary potential energies in deformational and configurational mechanics. Journal of Mechanics and Physics of Solids, 56, 772-800 (2008).

[34] Pao, Y. H. Electromagnetic forces in deformable continua, in Mechanics Today, vol. 4, pp. 209-305, ed. S. NematNasser, Pergamon, Oxford, 1978. 